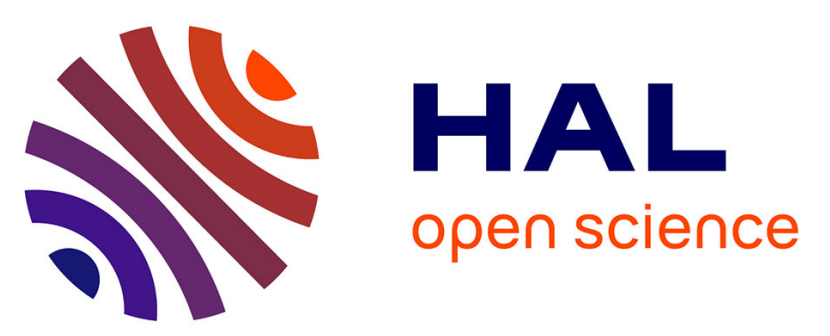

\title{
Compatibility conditions to allow some large amplitude WKB analysis for Burger's type systems
}

Christophe Cheverry, Mekki Houbad

\section{To cite this version:}

Christophe Cheverry, Mekki Houbad. Compatibility conditions to allow some large amplitude WKB analysis for Burger's type systems. Physica D: Nonlinear Phenomena, 2008, 237 (10-12), pp.1429-1443. hal-00148550

\section{HAL Id: hal-00148550 \\ https://hal.science/hal-00148550}

Submitted on 22 Nov 2007

HAL is a multi-disciplinary open access archive for the deposit and dissemination of scientific research documents, whether they are published or not. The documents may come from teaching and research institutions in France or abroad, or from public or private research centers.
L'archive ouverte pluridisciplinaire HAL, est destinée au dépôt et à la diffusion de documents scientifiques de niveau recherche, publiés ou non, émanant des établissements d'enseignement et de recherche français ou étrangers, des laboratoires publics ou privés. 


\title{
Compatibility conditions to allow some large amplitude WKB analysis for Burger's type systems
}

\author{
Christophe CHEVERRY ${ }^{1}$, Mekki HOUBAD ${ }^{2}$,
}

Abstract. Let $(T, \mathbf{V}, r) \in\left(\mathbb{R}_{+}^{*}\right)^{3}$. Work on the domain

$$
\Omega^{T}:=\left\{(t, x) \in[0, T] \times \mathbb{R}^{d} ;|x|+\mathbf{V} t \leq r\right\}, \quad d \in \mathbb{N} \backslash\{0,1\} .
$$

Consider the multidimensional Burger's type system

$$
\partial_{t} \mathbf{u}+\left(V \circ \mathbf{u} \cdot \nabla_{x}\right) \mathbf{u}=0, \quad \mathbf{u} \in \mathbb{R}^{d}, \quad(t, x) \in \Omega^{T}, \quad V \in \mathcal{C}^{1}\left(\mathbb{R}^{d} ; \mathbb{R}^{d}\right) .
$$

Associate (1) with a family of initial datas

$$
\left.\left.\mathbf{u}^{\varepsilon}(0, x)=h^{\varepsilon}(x)=H\left(x, \frac{\varphi(x)}{\varepsilon}\right)+O(\varepsilon), \quad \varepsilon \in\right] 0,1\right]
$$

defined on the ball $B(0, r]:=\left\{x \in \mathbb{R}^{d} ;|x| \leq r\right\}$, built with

$$
H(x, \theta) \in \mathcal{C}^{1}\left(B(0, r] \times \mathbb{T} ; \mathbb{R}^{d}\right), \quad \varphi(x) \in \mathcal{C}^{1}(B(0, r] ; \mathbb{R}), \quad \mathbb{T}:=\mathbb{R} / \mathbb{Z},
$$

and consisting of large amplitude high frequency monophase oscillating waves, which means to require

$$
\begin{array}{ll}
\exists(x, \theta) \in B(0, r] \times \mathbb{T} ; & \partial_{\theta} W(x, \theta) \neq 0, \quad W:=V \circ H, \\
\nabla_{x} \varphi(x) \neq 0, & \forall x \in B(0, r] .
\end{array}
$$

In general, due to the formation of shocks, the oscillating Cauchy problem (1) - (2) is not well-posed on the whole domain $\Omega^{T}$. In this article, we perform a detailed analysis of the restrictions to impose on the profile $H$ and on the phasis $\varphi$ in order to remedy this. Among these compatibility conditions, we isolate some new interesting system of nonlinear partial differential equations : see (1.2). We explain how to solve it and we describe how the underlying structure is propagated through the evolution equation (1).

\footnotetext{
${ }^{1}$ IRMAR, Université de Rennes I, Campus de Beaulieu, 35042 Rennes cedex, France, christophe.cheverry@univ-rennes1.fr.

${ }^{2}$ IRMAR, Université de Rennes I, Campus de Beaulieu, 35042 Rennes cedex, France, houbad.mekki@univ-rennes1.fr.
} 


\section{Contents}

1 Introduction. $\quad 2$

2 Analysis of the compatibility conditions $\quad 5$

3 Existence of compatible families $\quad 10$

4 Simple wave solutions $\quad 17$

\section{Introduction.}

Keep the notations and the hypothesis of the abstract. In particular

$$
x=\left(x_{1}, \cdots, x_{d}\right) \in \mathbb{R}^{d}, \quad|x|:=\left(\sum_{j=1}^{d} x_{j}^{2}\right)^{\frac{1}{2}}, \quad \partial_{j}:=\frac{\partial}{\partial x_{j}}, \quad \partial_{\theta}:=\frac{\partial}{\partial \theta} .
$$

To describe more precisely the expressions involved in (2), select a function

$$
\begin{aligned}
H:[0,1] \times B(0, r] \times \mathbb{T} & \longrightarrow \mathbb{R}^{d} \\
(\varepsilon, x, \theta) & \longmapsto H^{\varepsilon}(x, \theta)
\end{aligned}
$$

which is smooth with respect to the parameter $\varepsilon \in] 0,1]$

$$
H \in \mathcal{C}^{\infty}\left([0,1] ; \mathcal{C}^{1}\left(B(0, r] \times \mathbb{T} ; \mathbb{R}^{d}\right)\right)
$$

and whose Taylor expansion near $\varepsilon=0$ is noted

$$
H^{\varepsilon}(x, \theta)=H(x, \theta)+\sum_{j=1}^{m} \varepsilon^{j} H^{j}(x, \theta)+O\left(\varepsilon^{m+1}\right), \quad m \in \mathbb{N}^{*} .
$$

Let $\mathbf{u}={ }^{t}\left(\mathbf{u}_{1}, \cdots, \mathbf{u}_{d}\right) \in \mathbb{R}^{d}$. Note $\mathbf{u}^{\perp}$ or ${ }^{t} \mathbf{u}^{\perp}$ the hyperplane of $\mathbb{R}^{d}$ composed with the directions orthogonal to the vector $\mathbf{u}$, that is

$$
\mathbf{u}^{\perp} \equiv{ }^{t} \mathbf{u}^{\perp}:=\left\{\mathbf{v}={ }^{t}\left(\mathbf{v}_{1}, \cdots, \mathbf{v}_{d}\right) \in \mathbb{R}^{d} ;{ }^{t} \mathbf{v} \cdot \mathbf{u}=\sum_{j=1}^{d} \mathbf{v}_{j} \mathbf{u}_{j}=0\right\} .
$$

Introduce the orthogonal projector $\Pi_{F}$ from $\mathbb{R}^{d}$ onto the vector space $F$, that is the operator $\Pi_{F}$ defined by the conditions

$$
\mathbf{u}=\Pi_{F} \mathbf{u}+\left(I-\Pi_{F}\right) \mathbf{u}, \quad \Pi_{F} \mathbf{u} \in F, \quad\left(I-\Pi_{F}\right) \mathbf{u} \in F^{\perp} .
$$

Select some profile $W \in \mathcal{C}^{1}\left(B(0, r] \times \mathbb{T} ; \mathbb{R}^{d}\right)$. As usual, the symbol $D_{x} W(x, \theta)$ is for the Jacobian matrix

$$
D_{x} W(x, \theta)=\left(\partial_{j} W_{i}(x, \theta)\right)_{1 \leq i, j \leq d}, \quad W(x, \theta)={ }^{t}\left(W_{1}, \cdots, W_{d}\right) .
$$


Definition 1.1. The couple

$$
(\varphi, W) \in \mathcal{C}^{2}(B(0, r] ; \mathbb{R}) \times \mathcal{C}^{1}\left(B(0, r] \times \mathbb{T} ; \mathbb{R}^{d}\right)
$$

is said to be well prepared if it satisfies the following system

$$
\left\{\begin{array}{l}
\partial_{\theta} W(x, \theta) \subset \nabla \varphi(x)^{\perp} \\
\Pi_{\partial_{\theta} W(x, \theta)^{\perp}} D_{x} W(x, \theta) \Pi_{\nabla \varphi(x)^{\perp}}=0
\end{array}, \quad \forall(x, \theta) \in B(0, r] \times \mathbb{T} .\right.
$$

Define $h^{\varepsilon}(x):=H^{\varepsilon}\left(x, \frac{\varphi(x)}{\varepsilon}\right)$. Associate (1) with the family of initial datas $\left\{h^{\varepsilon}\right\}_{\varepsilon \in] 0,1]}$. The evolution equation (1) is a quasilinear (diagonal) system of hyperbolic equations. The speed of propagation is finite. More precisely, it can be uniformly controlled by

$$
\mathbb{R} \ni \mathbf{V}:=\left\{\sup \left|V \circ H^{\varepsilon}(x, \theta)\right| ;(\varepsilon, x, \theta) \in[0,1] \times B(0, r] \times \mathbb{T}\right\} .
$$

Standard results [5] guarantee the existence of $T^{\varepsilon}>0$ such that the Cauchy problem (1)-(2) has a local $\mathcal{C}^{1}$ solution $\mathbf{u}^{\varepsilon}(t, x)$ on the truncated cone $\Omega^{T^{\varepsilon}}$. In the context of (1), the limitations on $T^{\varepsilon}$ are due to the formation of shocks. The size of $T^{\varepsilon}$ can be estimated very precisely [1]-[3]-[6] in terms of $h^{\varepsilon}$. In general, this yields

$$
\limsup _{\varepsilon \longrightarrow 0} T^{\varepsilon}=0 \text {. }
$$

- In Section 2, we exhibit (Proposition 2.2) necessary and sufficient compatibility conditions on $\varphi(x)$ and $W(x, \theta):=V \circ H(x, \theta)$ in order to have

$$
\liminf _{\varepsilon \longrightarrow 0} T^{\varepsilon}=\bar{T}>0 .
$$

Among these compatibility conditions, we can isolate the system (1.2) whose study is in fact the main motivation of the present article. Indeed, the structure of (1.2) is new and interesting. It is not a usual quasilinear system because it is made of fully nonlinear constraints on the derivatives $\partial_{j} W_{i}$, $\partial_{\theta} W_{i}$ and $\partial_{j} \varphi$. It extends to the case $d \geq 3$ preliminary conditions which have been emphasized (only when $d=2$ ) in the recent contribution [3].

- In the Section 3, we classify all the solutions of (1.2). The fact that such a complete discussion is available is very surprising. At all events, this confirms the coherence of (1.2). The first observation is that any phasis $\varphi$ involved in (1.2) inherits some affine structure. Its level surfaces must be spanned by pieces of vector spaces (see Lemmas 3.2 and 3.3). This geometrical particularity seems to always play an important part when dealing with phasis involved in a supercritical WKB calculus, as here. 
Once $\varphi$ is determined, it becomes possible to identify all the profiles $W(x, \theta)$ which are subjected to (1.2). This is done in Proposition 3.1.

Quite a lot freedom is available in the construction of $W(x, \theta)$. The function $W(x, \theta)$ can be put in the form

$$
W(x, \theta)=W_{\|}(\varphi(x), \psi(x, \theta))+W_{\perp}(\varphi(x))
$$

where $W_{\|} \in \mathcal{C}^{1}\left(\mathbb{R}^{2} ; \mathbb{R}^{d}\right)$ and $W_{\perp} \in \mathcal{C}^{1}\left(\mathbb{R} ; \mathbb{R}^{d}\right)$ are conveniently well-polarized vector fields whereas $\psi \in \mathcal{C}^{1}(B(0, r] \times \mathbb{T} ; \mathbb{R})$ is any scalar function. Define

$$
\langle W\rangle(x) \equiv \bar{W}(x):=\int_{\mathbb{T}} W(x, \theta) d \theta, \quad W^{*}(x, \theta):=W(x, \theta)-\bar{W}(x) .
$$

The construction of large amplitude oscillating solutions to system (1) - or to variants of system (1) - is a delicate problem which has recently called some attention. The article [4] and the related contributions are mainly concerned with time oscillations. In the continuity of the works [1]-[2]-[3], we are faced here with the case of spatial oscillations.

According to Section 2, any family $\left\{h^{\varepsilon}\right\}_{\varepsilon} \in \mathcal{C}^{1}\left(B(0, r] ; \mathbb{R}^{d}\right)^{] 0,1]}$ issued from a well prepared couple $(\varphi, W)$ leads to a family $\left\{\mathbf{u}^{\varepsilon}\right\}_{\varepsilon}$ which is composed with $\mathcal{C}^{1}$ solutions $\mathbf{u}^{\varepsilon}$ of (1) on $\Omega^{\bar{T}}$. Now, the question is to determine the asymptotic behaviour of $\left\{\mathbf{u}^{\varepsilon}\right\}_{\varepsilon}$ when $\varepsilon$ goes to 0 . In particular, we want to understand how the constraint (1.2) is propagated through the evolution equation (1).

- Explanations are given in the Section 4. They can be obtained just by looking at the simple wave solutions of (1).

Theorem 1.1. Suppose that the couple

$$
(\varphi, W) \in \mathcal{C}^{2}(B(0, r] ; \mathbb{R}) \times \mathcal{C}^{1}\left(B(0, r] \times \mathbb{T} ; \mathbb{R}^{d}\right), \quad W:=V \circ H
$$

is well prepared. Then, the Cauchy problem consisting in the (apparently overdetermined) system

$$
\left\{\begin{array}{l}
\partial_{t} \mathbf{H}+V \circ \mathbf{H} \cdot \nabla_{x} \mathbf{H}=0, \\
\partial_{t} \Phi+\langle V \circ \mathbf{H}\rangle \cdot \nabla_{x} \Phi=0 \\
V \circ \mathbf{H}^{*} \cdot \nabla_{x} \Phi=0
\end{array}\right.
$$

associated with the initial datas

$$
\mathbf{H}(0, x, \theta)=H(x, \theta), \quad \Phi(0, x)=\varphi(x)
$$

has a unique solution on $\Omega^{T} \times \mathbb{T}$ for some $T>0$. For all $\left.\left.\varepsilon \in\right] 0,1\right]$, the simple wave $\mathbf{u}^{\varepsilon}(t, x):=\mathbf{H}\left(t, x, \frac{\Phi(t, x)}{\varepsilon}\right)$ is a solution of (1) on $\Omega^{T}$. Moreover, for all $t \in[0, T]$, the trace $(\Phi(t, \cdot), \mathbf{H}(t, \cdot))$ is still subjected to (1.2). 
At the time $t=0$, it is also possible to take into account (small) perturbations of $H\left(x, \frac{\varphi(x)}{\varepsilon}\right)$. For instance, we can select

$$
\left.\left.h^{\varepsilon}(x)=H^{\varepsilon}\left(x, \frac{\varphi(x)}{\varepsilon}\right), \quad \varepsilon \in\right] 0,1\right]
$$

where $H^{\varepsilon}(x, \theta)$ is like in (1.1). Again, the discussion of the Section 2 indicates that corresponding $\mathcal{C}^{1}$ solutions $\mathbf{u}^{\varepsilon}(t, x)$ of (1) are still available on $\Omega^{T}$. When $\varepsilon$ goes to 0 , the expression $\mathbf{u}^{\varepsilon}(t, x)$ remains closed (in a convenient sense) to the simple wave $\mathbf{H}\left(t, x, \frac{\Phi(t, x)}{\varepsilon}\right)$. This result can be proved by adapting and extending the method presented in [3]. The related analysis will not be developed here.

\section{Analysis of the compatibility conditions}

Introduce the curves $t \longmapsto(X(t ; x, \lambda), \Lambda(t ; x, \lambda))$ associated with the integration of (1) along the characteristics. They are defined by the ordinary differential equations

$$
\begin{cases}\frac{d}{d t} X=V(\Lambda), & X(0 ; x, \lambda)=x, \\ \frac{d}{d t} \Lambda=0, & \Lambda(0 ; x, \lambda)=\lambda .\end{cases}
$$

The corresponding solutions are

$$
X(t ; x, \lambda)=x+t V(\lambda), \quad \Lambda(t ; x, \lambda)=\lambda .
$$

Define

$$
\Phi^{\varepsilon}(t, x):=X\left(t ; x, h^{\varepsilon}(x)\right)=x+t W^{\varepsilon}\left(x, \frac{\varphi(x)}{\varepsilon}\right), \quad W^{\varepsilon}:=V \circ H^{\varepsilon} .
$$

Any smooth $\mathcal{C}^{1}$ solution of (1)-(2) must be subjected to the relation

$$
\mathbf{u}^{\varepsilon}\left(t, \Phi^{\varepsilon}(t, x)\right)=\mathbf{u}^{\varepsilon}\left(t, x+t V \circ h^{\varepsilon}(x)\right)=h^{\varepsilon}(x) .
$$

Fix $\varepsilon \in] 0,1]$. For $t$ small enough, say for $t \in\left[0, \tilde{T}^{\varepsilon}\right]$ with $\tilde{T}^{\varepsilon}>0$, the implicit theorem guarantees that the application

$$
\begin{aligned}
\Phi_{t}^{\varepsilon}: B(0, r] & \longrightarrow \Phi^{\varepsilon}(t, B(0, r]) \\
x & \longmapsto \Phi^{\varepsilon}(t, x)
\end{aligned}
$$

is a $\mathcal{C}^{1}$ diffeomorphism. Then, due to the definition of the maximal speed of propagation $\mathbf{V}$, any point $(t, x)$ contained in $\Omega^{\tilde{T}^{\varepsilon}}$ is sure to be realized as $(t, x)=\left(t, \Phi^{\varepsilon}(t, y)\right)$ for some unique $y \in B(0, r]$. We can define

$$
\mathbf{u}^{\varepsilon}(t, x):=h^{\varepsilon} \circ\left(\Phi_{t}^{\varepsilon}\right)^{-1}(x), \quad(t, x) \in \Omega^{\tilde{T}^{\varepsilon}}
$$


which yields a $\mathcal{C}^{1}$ solution on $\Omega^{\tilde{T}^{\varepsilon}}$ of the Cauchy problem (1)-(2). The relation (2.4) implies that

$$
D_{x} \mathbf{u}^{\varepsilon}(t, x):=D_{x} h^{\varepsilon} \circ\left(\Phi_{t}^{\varepsilon}\right)^{-1}(x) \operatorname{Co}\left[D_{x} \Phi^{\varepsilon}(t, x)\right] / \operatorname{det} D_{x} \Phi^{\varepsilon}(t, x)
$$

where $\mathrm{Co}[M]$ stands for the co-matrix of $M$. We have

$$
\begin{aligned}
D_{x} \Phi^{\varepsilon}(t, x)= & \varepsilon^{-1} t \partial_{\theta} W^{\varepsilon}\left(x, \frac{\varphi(x)}{\varepsilon}\right) \otimes{ }^{t} \nabla \varphi(x) \\
& +\mathrm{I}+t D_{x} W^{\varepsilon}\left(x, \frac{\varphi(x)}{\varepsilon}\right)
\end{aligned}
$$

where we adopt the following convention

$$
u \otimes v=\left(u_{i} v_{j}\right)_{1 \leq i, j \leq d}, \quad u={ }^{t}\left(u_{1}, \cdots, u_{d}\right), \quad v={ }^{t}\left(v_{1}, \cdots, v_{d}\right) .
$$

Classical results - see for instance [5] - assert that a $\mathcal{C}^{1}$ solution $\mathbf{u}^{\varepsilon}(t, x)$ on $\Omega^{T}$ can be extended in time as long as the matrix $D_{x} \mathbf{u}^{\varepsilon}(t, x)$ is bounded. In view of the formula (2.5), to recover a $\mathcal{C}^{1}$ solution $\mathbf{u}^{\varepsilon}(t, x)$ on $\Omega^{T}$, it is necessary and sufficient to have

$$
\operatorname{det} D_{x} \Phi^{\varepsilon}(t, x)>0, \quad \forall(t, x) \in \Omega^{T} .
$$

Therefore, the life span of a $\mathcal{C}^{1}$ solution on a domain of propagation is bounded below by

$$
T^{\varepsilon}:=\sup \left\{T>0 ; \operatorname{det} D_{x} \Phi^{\varepsilon}(t, x)>0, \forall(t, x) \in[0, T] \times B(0, r[\} .\right.
$$

In general, due to the presence in (2.6) of the (singular) term with $\varepsilon^{-1}$ in factor, only (1.3) can be asserted. Now, the opposite situation is still possible providing that the family $\left\{h^{\varepsilon}\right\}_{\varepsilon}$ is conveniently adjusted. This situation is distinguished below.

Definition 2.1. The family $\left\{h^{\varepsilon}\right\}_{\varepsilon}$ is said to be compatible if there exists $T>0$ and $c>0$ such that

$$
\left.\left.\operatorname{det} D_{x} \Phi^{\varepsilon}(t, x) \geq c>0, \quad \forall(t, x, \varepsilon) \in[0, T] \times B(0, r] \times\right] 0,1\right] .
$$

The preceding discussion can be summarized by the following statement.

Proposition 2.1. Suppose that the family $\left\{h^{\varepsilon}\right\}_{\varepsilon}$ is compatible. Then, for all $\varepsilon \in] 0,1]$, the expression $\mathbf{u}^{\varepsilon}(t, x)$ defined through (2.4) is a $\mathcal{C}^{1}$ solution on $\Omega^{T}$ of the Cauchy problem (1)-(2).

Our aim now is to transcribe (2.7) in terms of constraints to impose on $\varphi(x)$ and $W(x, \theta)$. To this end, introduce

$$
\mathcal{V}^{*}:=\left\{(x, \theta) \in B(0, r] \times \mathbb{T} ; \partial_{\theta} W(x, \theta) \neq 0\right\} .
$$

We assume (3), that is $\mathcal{V}^{*} \neq \emptyset$. 
Proposition 2.2. The family $\left\{h^{\varepsilon}\right\}_{\varepsilon}$ is compatible if and only if the two following conditions are satisfied.

i) The profile $W^{*}$ is polarized according to :

$$
{ }^{t} \nabla \varphi(x) \cdot \partial_{\theta} W^{*}(x, \theta)=0, \quad \forall(x, \theta) \in \mathcal{V}^{*} .
$$

ii) There is $T>0$ such that for all $(t, x, \theta) \in[0, T] \times B(0, r] \times \mathbb{T}$, we have:

$$
(-1)^{d} \operatorname{det}\left[\Pi_{\partial_{\theta} W(x, \theta)^{\perp}}\left(\mathrm{I}+t D_{x} W(x, \theta)\right) \Pi_{\nabla \varphi(x)^{\perp}}\right] \geq 0 .
$$

Proof. The reasoning is based on the identity (2.6) which can also be formulated as

$$
\varepsilon D_{x} \Phi^{\varepsilon}(t, x)=M^{0}\left(t, x, \frac{\varphi(x)}{\varepsilon}\right)+\varepsilon M^{1}\left(t, x, \frac{\varphi(x)}{\varepsilon}\right)+\varepsilon^{2} t R^{\varepsilon}\left(t, x, \frac{\varphi(x)}{\varepsilon}\right)
$$

where

$$
\begin{aligned}
& M^{0}(t, x, \theta):=t \partial_{\theta} W(x, \theta) \otimes{ }^{t} \nabla \varphi(x), \\
& M^{1}(t, x, \theta):=\mathrm{I}+t D_{x} W(x, \theta)+t \partial_{\theta}\left[D_{\mathbf{u}} V\left(H^{0}(x, \theta)\right) H^{1}(x, \theta)\right] \otimes{ }^{t} \nabla \varphi(x),
\end{aligned}
$$

whereas the matrix $R^{\varepsilon}(t, x, \theta)$ is a continuous function of all the variables $(\varepsilon, t, x, \theta) \in[0,1] \times \mathbb{R} \times B(0, r] \times \mathbb{T}$.

- a $\bullet$ About the constraint (2.9). Assume that the restriction (2.7) is satisfied for some $T>0$ and some $c>0$. We start by showing

$$
{ }^{t} \nabla \varphi(x) \cdot \partial_{\theta} W^{*}(x, \theta) \geq 0, \quad \forall(x, \theta) \in \mathcal{V}^{*} .
$$

To this end, we argue by contradiction. We suppose that we can find a point $(\bar{x}, \bar{\theta}) \in \mathcal{V}^{*}$ such that

$$
{ }^{t} \nabla \varphi(\bar{x}) \cdot \partial_{\theta} W^{*}(\bar{x}, \bar{\theta})<0 .
$$

This information allows to express the matrices $M^{0}(t, \bar{x}, \bar{\theta})$ and $M^{1}(t, \bar{x}, \bar{\theta})$ in a basis of $\mathbb{R}^{d}$ having the form $\left(e_{1}, e_{2}, \cdots, e_{d}\right)$ where $e_{1}:=\partial_{\theta} W(\bar{x}, \bar{\theta})$ and where $\left(e_{2}, \cdots, e_{d}\right)$ is a basis of $\nabla \varphi(\bar{x})^{\perp}$. In this special basis, the matrices $M^{0}$ and $M^{1}$ look like

$$
M^{0}=\left(\begin{array}{cccc}
{ }^{t} \nabla \varphi \cdot \partial_{\theta} W & 0 & \ldots & 0 \\
0 & 0 & \ldots & 0 \\
\vdots & \vdots & & \vdots \\
0 & 0 & \cdots & 0
\end{array}\right), \quad M^{1}=\left(\begin{array}{ccc}
m_{11}^{1} & \ldots & m_{1 d}^{1} \\
m_{21}^{1} & \ldots & m_{2 d}^{1} \\
\vdots & & \vdots \\
m_{d 1}^{1} & \ldots & m_{d d}^{1}
\end{array}\right) .
$$

It follows that 


$$
\begin{aligned}
\operatorname{det} D_{x} \Phi^{\varepsilon}(t, \bar{x}) & =\varepsilon^{-d} \operatorname{det}\left[M^{0}\left(t, \bar{x}, \frac{\varphi(\bar{x})}{\varepsilon}\right)+\varepsilon M^{1}\left(t, \bar{x}, \frac{\varphi(\bar{x})}{\varepsilon}\right)+O\left(\varepsilon^{2}\right)\right] \\
& =\varepsilon^{-1} t^{t} \nabla \varphi(\bar{x}) \cdot \partial_{\theta} W\left(\bar{x}, \frac{\varphi(\bar{x})}{\varepsilon}\right) \operatorname{det} M^{b}\left(t, \bar{x}, \frac{\varphi(\bar{x})}{\varepsilon}\right)+O(1)
\end{aligned}
$$

with

$$
M^{b}=M^{b}(t, \bar{x}, \bar{\theta})=\left(\begin{array}{ccc}
m_{22}^{1} & \ldots & m_{2 d}^{1} \\
\vdots & & \vdots \\
m_{d 2}^{1} & \ldots & m_{d d}^{1}
\end{array}\right) .
$$

When $t=0$, we have $M^{1}(0, \bar{x}, \bar{\theta})=\mathrm{I}$ so that $M^{b}=\mathrm{I}_{\mathbb{R}^{d-1}}$ and $\operatorname{det} M^{b}=1$. By continuity, for $t$ small enough (say $t \in[0, \tilde{T}]$ with $\tilde{T}>0$ ), it remains

$$
\left.\left.\operatorname{det} M^{b}\left(t, \bar{x}, \frac{\varphi(\bar{x})}{\varepsilon}\right) \geq \frac{1}{2}, \quad \forall(t, \varepsilon) \in[0, \tilde{T}] \times\right] 0,1\right] .
$$

Choose $t \in] 0, \tilde{T}]$ and a sequence $\left.\left.\left\{\varepsilon_{n}\right\}_{n} \in\right] 0,1\right]^{\mathbb{N}}$ tending to 0 and such that

$$
\forall n \in \mathbb{N}, \quad \exists k_{n} \in \mathbb{Z} ; \quad \varphi(\bar{x})=\varepsilon_{n}\left(\bar{\theta}+2 k_{n} \pi\right) .
$$

Then, by construction, we have

$$
\exists C \in \mathbb{R} ; \quad \operatorname{det} D_{x} \Phi^{\varepsilon_{n}}(t, \bar{x}) \leq \frac{t}{2 \varepsilon_{n}}{ }^{t} \nabla \varphi(\bar{x}) \cdot \partial_{\theta} W(\bar{x}, \bar{\theta})+C, \quad \forall n \in \mathbb{N} .
$$

For $n$ large enough, the right hand side becomes negative. This is not compatible with (2.7). This means that the case (2.12) must be excluded. Now, because the function $\theta \longmapsto W(x, \theta)$ is periodic, we have

$$
\int_{0}^{1}{ }^{t} \nabla \varphi(x) \cdot \partial_{\theta} W(x, \theta) d \theta={ }^{t} \nabla \varphi(x) \cdot W(x, \theta+1)-{ }^{t} \nabla \varphi(x) \cdot W(x, \theta)=0 .
$$

Combining this with (2.11), we see that the restriction (2.9) is necessary.

- b - About the constraint (2.10). Assume again that the restriction (2.7) is satisfied for some $T>0$ and some $c>0$. We already know that (2.9) is verified. This information allows to work in a basis $\left(\tilde{e}_{1}, \cdots, \tilde{e}_{d}\right)$ of $\mathbb{R}^{d}$ adjusted such that $\tilde{e}_{d}:={ }^{t} \nabla \varphi(x)$ whereas $\left(\tilde{e}_{1}, \cdots, \tilde{e}_{d-1}\right)$ is a basis of $\nabla \varphi(x)^{\perp}$ with $\tilde{e}_{1}:=\partial_{\theta} W(x, \theta)$. In this new special basis, the matrices $M^{0}$ and $M^{1}$ take the form

$$
M^{0}=\left(\begin{array}{cccc}
0 & \cdots & 0 & t|\nabla \varphi|^{2} \\
0 & \cdots & 0 & 0 \\
\vdots & & \vdots & \vdots \\
0 & \cdots & 0 & 0
\end{array}\right), \quad M^{1}=\left(\begin{array}{ccc}
m_{11}^{1} & \ldots & m_{1 d}^{1} \\
m_{21}^{1} & \cdots & m_{2 d}^{1} \\
\vdots & & \vdots \\
m_{d 1}^{1} & \cdots & m_{d d}^{1}
\end{array}\right) .
$$

It follows that

$$
\begin{aligned}
\operatorname{det} D_{x} \Phi^{\varepsilon}(t, x)= & \varepsilon^{-1}(-1)^{d} t|\nabla \varphi(x)|^{2} \operatorname{det} M^{\sharp}\left(t, x, \frac{\varphi(x)}{\varepsilon}\right) \\
& +1+t g^{\varepsilon}\left(t, x, \frac{\varphi(x)}{\varepsilon}\right)
\end{aligned}
$$


with

$$
M^{\sharp}(t, x, \theta)=\left(\begin{array}{ccc}
m_{21}^{1} & \ldots & m_{2(d-1)}^{1} \\
\vdots & & \vdots \\
m_{d 1}^{1} & \cdots & m_{d(d-1)}^{1}
\end{array}\right)=\Pi_{\partial_{\theta} W(x, \theta)^{\perp}} M^{1} \Pi_{\nabla \varphi(x)^{\perp}}
$$

whereas the scalar application $g^{\varepsilon}(t, x, \theta)$ is a continuous function of all the variables $(\varepsilon, t, x, \theta) \in[0,1] \times \mathbb{R} \times B(0, r] \times \mathbb{T}$. Observe that

$$
\left[\mathbf{u} \otimes{ }^{t} \nabla \varphi(x)\right] \mathbf{v}=0, \quad \forall(\mathbf{u}, \mathbf{v}) \in \mathbb{R}^{d} \times \nabla \varphi(x)^{\perp} .
$$

Therefore, the expression of $M^{\sharp}$ can be simplified according to

$$
M^{\sharp}(t, x, \theta)=\Pi_{\partial_{\theta} W(x, \theta)^{\perp}}\left(\mathrm{I}+t D_{x} W(x, \theta)\right) \Pi_{\nabla \varphi(x)^{\perp}} .
$$

Follow the argument of the paragraph a, using a well adjusted sequence $\left\{\varepsilon_{n}\right\}_{n}$, in order to extract the necessary condition

$$
(-1)^{d} \operatorname{det} M^{\sharp}(t, x, \theta) \geq 0, \quad \forall(t, x, \theta) \in[0, T] \times B(0, r] \times \mathbb{T}
$$

which is exactly (2.10).

- c • Sufficient conditions. Conversely, suppose (2.9) and (2.10). Because of (2.9), we can use the basis $\left(\tilde{e}_{1}, \ldots, \tilde{e}_{d}\right)$. The computations of the paragraph $\mathbf{b}$ remain valid. Because of the sign condition (2.10), we are sure that

$$
\left.\left.\operatorname{det} D_{x} \Phi^{\varepsilon}(t, x) \geq 1-C t, \quad \forall(t, x, \varepsilon) \in[0, T] \times B(0, r] \times\right] 0,1\right]
$$

for some $C>0$. It suffices to choose $T>0$ small enough to recover (2.7).

Remark 2.1. Suppose that $V: \mathbb{R}^{d} \longrightarrow \mathbb{R}^{d}$ is a $\mathcal{C}^{1}$ diffeomorphism. Then, it is equivalent to solve (1) or

$$
\partial_{t} \mathbf{w}+\left(\mathbf{w} \cdot \nabla_{x}\right) \mathbf{w}=0, \quad \mathbf{w}:=V \circ \mathbf{u}
$$

completed with the initial data

$$
\left.\left.\mathbf{w}(0, x)=W\left(x, \frac{\varphi(x)}{\varepsilon}\right), \quad \varepsilon \in\right] 0,1\right] .
$$

The system (1.2) can also be interpreted as a compatibility condition in order to solve the Cauchy problem (2.13)-(2.14) in the class of $\mathcal{C}^{1}$ solutions, locally in time, on some domain $\Omega^{T}$ with $T>0$ independent of $\left.\left.\varepsilon \in\right] 0,1\right]$. This interpretation explains why the relevant constraint is concerned with $V \circ H$ instead of dealing separately with $V$ and $H$.

Remark 2.2. When $d \gg 1$, the condition ii) of Proposition 2.2 can be difficult to deal with. In the basis $\left(\tilde{e}_{1}, \cdots, \tilde{e}_{d}\right)$, we get 


$$
M^{\sharp}(t, x, \theta)=\left(\begin{array}{cccccc}
0 & 1 & 0 & \cdots & 0 & 0 \\
0 & 0 & 1 & \cdots & 0 & 0 \\
\vdots & \vdots & \vdots & & \vdots & \vdots \\
0 & 0 & 0 & \cdots & 0 & 1 \\
0 & 0 & 0 & \cdots & 0 & 0
\end{array}\right)+t \tilde{M}^{\sharp}(x, \theta) .
$$

This special structure implies the existence of coefficients $\alpha_{j}(x, \theta)$ such that

$$
(-1)^{d} \operatorname{det} M^{\sharp}(t, x, \theta)=\sum_{j=1}^{d-1} \alpha_{j}(x, \theta) t^{j} .
$$

Noting

$$
J(x, \theta):=\left\{\begin{array}{lll}
\min \mathcal{J} & \text { if } \mathcal{J}:=\left\{j ; \alpha_{j}(x, \theta) \neq 0\right\} \neq \emptyset \\
d-1 & \text { if } \mathcal{J}=\emptyset
\end{array}\right.
$$

the condition (2.10) is equivalent to the restriction

$$
\alpha_{J(x, \theta)}(x, \theta) \geq 0, \quad \forall(x, \theta) \in B(0, r] \times \mathbb{T} .
$$

In what follows, instead of checking (2.15), we will be satisfied with the more restrictive condition

$$
\Pi_{\partial_{\theta} W(x, \theta)^{\perp}} D_{x} W(x, \theta) \Pi_{\nabla \varphi(x)^{\perp}}=0 .
$$

In other words, we concentrate on well prepared couples $(\varphi, W)$.

\section{Existence of compatible families}

The goal of this subsection is to show through a constructive proof that the system (1.2) actually admits solutions, and to understand the structure of these solutions.

Consider $\varphi \in \mathcal{C}^{2}(B(0, r] ; \mathbb{R})$ with no critical point in $B(0, r]$. Without loss of generality (relabelling the coordinates and diminishing $r$ if necessary) we can adjust $\varphi$ so that

$$
\partial_{d} \varphi(x) \neq 0, \quad \forall x \in B(0, r], \quad r>0 .
$$

Introduce the linear subspace

$$
\mathbf{E}(x):=\left\{W^{*}(x, \theta) ; \theta \in \mathbb{T}\right\} \subset \mathbb{R}^{d}, \quad W \in \mathcal{C}^{1}\left(B(0, r] \times \mathbb{T}, \mathbb{R}^{d}\right) .
$$


For the sake of simplicity, we will restrict the analysis to the case where the application $x \longmapsto \mathbf{E}(x)$ is constant on $B(0, r]$ :

$$
\exists J \in\{1, \cdots, d-1\} ; \quad \operatorname{dim} \mathbf{E}(x)=J, \quad \forall x \in B(0, r] .
$$

Denote by $\mathcal{G}_{d}^{J}$ the Grassmanian manifold of linear subspaces of $\mathbb{R}^{d}$ with dimension $J$.

Lemma 3.1. Assume (3.2) and (3.3). Then $\mathbf{E} \in \mathcal{C}^{1}\left(B(0, r], \mathcal{G}_{d}^{J}\right)$.

Proof. Let $x_{0} \in B(0, r]$. By hypothesis, we can find $\theta_{1}, \cdots, \theta_{J}$ in $\mathbb{T}$ such that $\left(W^{*}\left(x_{0}, \theta_{1}\right), \cdots, W^{*}\left(x_{0}, \theta_{J}\right)\right)$ is a basis of $\mathbf{E}\left(x_{0}\right)$. Hence, we can extract a $J \times J$ determinant

$$
\delta\left(x_{0}\right):=\operatorname{det}\left(W_{i_{j}}^{*}\left(x_{0}, \theta_{k}\right)\right)_{1 \leq j, k \leq J}, \quad i_{j} \in \llbracket 1, d \rrbracket
$$

such that $\delta\left(x_{0}\right) \neq 0$. Since $W^{*}$ is continuous, the function $x \longmapsto \delta(x)$ is continuous. Therefore, we can isolate some small open neighborhood $\Omega$ of $x_{0}$ such that

$$
\delta(x) \neq 0, \quad \forall x \in \Omega, \quad x_{0} \in \Omega .
$$

This means that $\left(W^{*}\left(\cdot, \theta_{1}\right), \cdots, W^{*}\left(\cdot, \theta_{J}\right)\right)$ is a $\mathcal{C}^{1}$ basis of $\mathbf{E}$ in $\Omega$. Since $x_{0} \in B(0, r]$ can be chosen arbitrarily, this proves the Lemma 3.1.

Now, it is clear that

$$
\partial_{\theta} W^{*}(x, \theta) \subset \mathbf{E}(x) \subset \nabla \varphi(x)^{\perp}, \quad \forall(x, \theta) \in B(0, r] \times \mathbb{T} .
$$

Therefore, the condition (2.16) implies that

$$
\Pi_{\mathbf{E}(x)^{\perp}} D_{x} W^{*}(x, \theta) \Pi_{\nabla \varphi(x)^{\perp}}=0, \quad \forall(x, \theta) \in B(0, r] \times \mathbb{T} .
$$

Lemma 3.2. Let $\varphi \in \mathcal{C}^{2}(B(0, r], \mathbb{R})$ and $W \in \mathcal{C}^{1}\left(B(0, r] \times \mathbb{T}, \mathbb{R}^{d}\right)$ satisfying respectively the conditions (3.1) and (3.3). Suppose that the relations (3.4) and (3.5) are satisfied. Then, the application $x \longmapsto \mathbf{E}(x)$ is constant on the level surfaces of $\varphi$. More precisely

$$
\exists \mathbb{E} \in \mathcal{C}^{1}\left(\mathbb{R}, \mathcal{G}_{d}^{J}\right) ; \quad \mathbf{E}(x)=\mathbb{E} \circ \varphi(x), \quad \forall x \in B(0, r] .
$$

Proof. Let us denote $\delta_{i j}$ the usual Dirichlet symbol, and $\delta^{(k)}$ the vector of $\mathbb{R}^{d}$ whose components are $\left(\delta_{i k}\right)_{1 \leq i \leq d}$. The $d-1$ vectors 


$$
v_{k}(x)=-\delta^{(k)}+\partial_{k} \varphi(x) / \partial_{d} \varphi(x) \delta^{(d)}, \quad 1 \leq k \leq d-1
$$

form a $\mathcal{C}^{1}$ basis of $\nabla \varphi(x)^{\perp}$. By permutting the components of $\mathbb{R}^{d}$ and by diminishing $r$ if necessary, we can always arrange the datas so that

$$
\mathbf{E}(x) \oplus\left\langle v_{1}(x), \cdots, v_{d-J-1}(x)\right\rangle=\nabla \varphi(x)^{\perp}, \quad \forall x \in B(0, r] .
$$

Therefore, for all $j \in \llbracket 1, J \rrbracket$, the vector $v_{d-j}(x) \in \nabla \varphi(x)^{\perp}$ can be decomposed according to

$$
v_{d-j}(x)=e_{j}(x)-\sum_{k=1}^{d-J-1} \alpha_{j}^{k}(x) v_{k}(x), \quad e_{j}(x) \in \mathbf{E}(x)
$$

where, due to the assumptions related to the regularity of $\varphi$ and $\mathbf{E}$, we have

$$
e_{j}=\left(e_{j}^{1}, \cdots, e_{j}^{d}\right) \in \mathcal{C}^{1}\left(B(0, r] ; \mathbb{R}^{d}\right), \quad \alpha_{j}^{k} \in \mathcal{C}^{1}(B(0, r] ; \mathbb{R}) .
$$

The vectors $e_{j}$ with $j \in \llbracket 1, J \rrbracket$ are necessarily independent. They form a basis of $\mathbf{E}(x)$. In view of (3.4), the profile $W$ writes

$$
W(x, \theta)=\bar{W}(x)+\sum_{j=1}^{J} w_{j}^{*}(x, \theta) e_{j}(x), \quad w_{j}^{*} \in \mathcal{C}^{1}(B(0, r] \times \mathbb{T} ; \mathbb{R})
$$

and the relation $(3.5)$ reads

$$
\sum_{j=1}^{J} w_{j}^{*}(x, \theta) \Pi_{\mathbf{E}(x)^{\perp}} D_{x} e_{j}(x) \Pi_{\nabla \varphi(x)^{\perp}}=0, \quad \forall(x, \theta) \in B(0, r] \times \mathbb{T} .
$$

Recall that the dimension of $\mathbf{E}(x)$ is $J$. This implies that

$$
\exists\left(\theta_{1}^{x}, \cdots, \theta_{J}^{x}\right) \in \mathbb{T}^{J} ; \quad \operatorname{det}\left[w_{i}^{*}\left(x, \theta_{j}^{x}\right)\right]_{1 \leq i, j \leq J} \neq 0 .
$$

Combining the preceding informations, we see that the relation (3.5) is equivalent to

$$
\Pi_{\mathbf{E}(x)^{\perp}} D_{x} e_{j}(x) \Pi_{\nabla \varphi(x)^{\perp}}=0, \quad \forall(j, x) \in \llbracket 1, J \rrbracket \times B(0, r] .
$$

The vector space $\mathbf{E}(x)^{\perp}$ is of dimension $d-J$. It is generated by the vector $e_{d}(x):=\nabla \varphi(x)$ and the $d-J-1$ vectors

$$
e_{j}(x)=-\delta^{(j-J)}+\sum_{k=1}^{J} \alpha_{k}^{j-J}(x) \delta^{(d-k)}, \quad j \in \llbracket J+1, d-1 \rrbracket .
$$

Therefore (3.7) is exactly the same as

$$
{ }^{t} e_{l}(x) D_{x} e_{j}(x) \Pi_{\nabla \varphi(x)^{\perp}}=0, \quad \forall(l, j, x) \in \llbracket J+1, d \rrbracket \times \llbracket 1, J \rrbracket \times B(0, r] .
$$

For $j \in \llbracket 1, J \rrbracket$, compute

$$
D_{x} e_{j}(x)=\sum_{k=1}^{d-J-1} \nabla_{x} \alpha_{j}^{k}(x) v_{k}(x)
$$




$$
+\left[\sum_{k=1}^{d-J-1} \alpha_{j}^{k}(x) \nabla_{x}\left(\partial_{k} \varphi(x) / \partial_{d} \varphi(x)\right)+\nabla_{x}\left(\partial_{d-j} \varphi(x) / \partial_{d} \varphi(x)\right)\right] \delta^{(d)} .
$$

Applying on the left ${ }^{t} e_{l}(x)$ with $l \in \llbracket J+1, d-1 \rrbracket$, yields

$$
{ }^{t} e_{l}(x) D_{x} e_{j}(x)=\nabla_{x} \alpha_{j}^{l-J}(x), \quad 1 \leq j \leq J<l \leq d-1 .
$$

We can extract from (3.8) that

$$
\nabla_{x} \alpha_{j}^{l-J}(x) \Pi_{\nabla \varphi(x)^{\perp}}=0, \quad \forall(l, j, x) \in \llbracket J+1, d-1 \rrbracket \times \llbracket 1, J \rrbracket \times B(0, r]
$$

which means that the scalar functions $\alpha_{j}^{l-J}$ and $\varphi$ have common level surfaces. In other words, we can find

$$
Z_{j}^{k} \in \mathcal{C}^{1}(\mathbb{R}, \mathbb{R}), \quad(k, j) \in \llbracket 1, d-J-1 \rrbracket \times \llbracket 1, J \rrbracket
$$

such that, for all $(k, j) \in \llbracket 1, d-J-1 \rrbracket \times \llbracket 1, J \rrbracket$, we have

$$
e_{j}^{k}(x)=-\alpha_{j}^{k}(x)=Z_{j}^{k} \circ \varphi(x), \quad \forall x \in B(0, r] .
$$

The last conditions to consider are obtained by taking $j \in \llbracket 1, J \rrbracket$ and $l=d$. Namely

$$
\nabla \varphi(x) D_{x} e_{j}(x) \Pi_{\nabla \varphi(x)^{\perp}}=0, \quad \forall(j, x) \in \llbracket 1, J \rrbracket \times B(0, r] .
$$

Use (3.1) and (3.9) to simplify this into

$$
\nabla_{x} e_{j}^{d}(x) \Pi_{\nabla \varphi(x)^{\perp}}=0, \quad \forall(j, x) \in \llbracket 1, J \rrbracket \times B(0, r]
$$

where we recall that

$$
e_{j}^{d}(x)=-\sum_{k=1}^{d-J-1} Z_{j}^{k} \circ \varphi(x) \partial_{k} \varphi(x) / \partial_{d} \varphi(x)+\partial_{d-j} \varphi(x) / \partial_{d} \varphi(x) .
$$

Again, this means the existence of $Z_{j}^{d} \in \mathcal{C}^{1}(\mathbb{R}, \mathbb{R})$ such that

$$
e_{j}^{d}(x)=Z_{j}^{d} \circ \varphi(x), \quad \forall(j, x) \in \llbracket 1, J \rrbracket \times B(0, r] .
$$

Briefly, we have obtained, for all $j \in \llbracket 1, J \rrbracket$, that

$$
e_{j}(x)=Z_{j} \circ \varphi(x), \quad Z_{j}={ }^{t}\left(Z_{j}^{1}, \cdots, Z_{j}^{d-J-1}, 0, \cdots, 0,-1,0, \cdots, 0, Z_{j}^{d}\right) .
$$

The vector space $\mathbf{E}$ is spanned by the $e_{j}$ with $j \in \llbracket 1, J \rrbracket$. Therefore, it depends only on $\varphi$, in a $\mathcal{C}^{1}$ way. This gives rise to (3.6). In particular, $\mathbf{E}$ is constant on the level surfaces of $\varphi$.

Combining (3.4) and (3.6), we can produce the necessary condition

$$
\nabla \varphi(x) \in \mathbb{E} \circ \varphi(x)^{\perp}=\mathbf{E}(x)^{\perp}, \quad \forall x \in B(0, r] .
$$


The condition (3.10) is a geometrical constraint on $\varphi$ underlying the resolution of (1.2). We explain below how to solve it.

Lemma 3.3. Select :

- a curve $\mathbb{E} \in \mathcal{C}^{2}\left(\mathbb{R}, \mathcal{G}_{d}^{J}\right)$ of J-dimensional vector spaces of $\mathbb{R}^{d}$,

- a $\mathcal{C}^{2}$ submanifold $\mathcal{S} \subset \mathbb{R}^{d}$ of dimension $d-J$, containing $0 \in \mathbb{R}^{d}$,

- a $\mathcal{C}^{2}$ scalar function $\chi: \mathcal{S} \longrightarrow \mathbb{R}$.

Note $T_{0} \mathcal{S}$ the tangent space of $\mathcal{S}$ at the point $0 \in \mathbb{R}^{d}$. We suppose that

$$
T_{0} \mathcal{S}+\mathbb{E}(\chi(0))=\mathbb{R}^{d} .
$$

Then, we can find $r>0$ such that the nonlinear equation (3.10) completed with $\varphi_{\mid \mathcal{S} \cap B(0, r]} \equiv \chi$ has a unique $\mathcal{C}^{2}$ solution on $B(0, r]$. We will say that the phase $\varphi$ is generated by $(\mathbb{E}, \mathcal{S}, \chi)$.

Proof. Select $\delta>0$ and $J$ functions

$$
Z_{j} \in \mathcal{C}^{2}(] \chi(0)-\delta, \chi(0)+\delta[; \mathbb{R}), \quad j \in \llbracket 1, J \rrbracket
$$

adjusted such that, for all $t \in] \chi(0)-\delta, \chi(0)+\delta\left[,\left(Z_{1}(t), \cdots, Z_{J}(t)\right)\right.$ is a basis of $\mathbb{E}(t)$. Note

$$
\Omega_{\mathcal{S}}^{\delta}:=\chi^{-1}(] \chi(0)-\delta, \chi(0)+\delta[) \subset \mathcal{S}, \quad z={ }^{t}\left(z^{1}, \ldots, z^{J}\right) \in \mathbb{R}^{J} .
$$

Consider the $\mathcal{C}^{2}$ application

$$
\begin{aligned}
\Xi: \Omega_{\mathcal{S}}^{\delta} \times \mathbb{R}^{J} & \longrightarrow \mathbb{R}^{d} \\
(y, z) & \longmapsto \Xi(y, z):=y+\sum_{j=1}^{J} z^{j} Z_{j} \circ \chi(y) .
\end{aligned}
$$

Because of (3.11), the linear operator

$$
\begin{aligned}
D_{x} \Xi(0,0): T_{0} \mathcal{S} \times \mathbb{R}^{J} & \longrightarrow \mathbb{R}^{d} \\
(h, k) & \longmapsto h+\sum_{j=1}^{J} k^{j} Z_{j} \circ \chi(y)
\end{aligned}
$$

is invertible. The inverse mapping Theorem can be applied at the point $(0,0) \in \mathcal{S} \times \mathbb{R}^{J}$. It guarantees the existence of $r>0$ such that $\Xi$ is a $\mathcal{C}^{2}$ diffeomorphism from a neighbourhood of $(0,0) \in \mathcal{S} \times \mathbb{R}^{J}$ onto $B(0, r]$. Introduce the projection

$$
\begin{aligned}
\Gamma: \mathcal{S} \times \mathbb{R}^{J} & \longrightarrow \mathcal{S} \\
(y, z) & \longmapsto \Gamma(y, z):=y .
\end{aligned}
$$

Now, we can define

$$
\varphi:=\chi \circ \Gamma \circ \Xi^{-1} \in \mathcal{C}^{2}(B(0, r] ; \mathbb{R}) .
$$


Since $\left(\Gamma \circ \Xi^{-1}\right)_{\mid \mathcal{S} \cap B(0, r]}=I d$, we have $\varphi_{\mid \mathcal{S} \cap B(0, r]} \equiv \chi_{\mid \mathcal{S} \cap B(0, r]}$. Moreover, the function $\varphi$ is constant on the set

$$
\mathcal{F}_{y}:=\left(y+\left\langle Z_{1} \circ \chi(y), \cdots, Z_{J} \circ \chi(y)\right\rangle\right) \cap B(0, r], \quad y \in \mathcal{S} \cap B(0, r] .
$$

More precisely, $\mathcal{F}_{y}$ is a piece of affine manifold with direction $\mathbb{E} \circ \chi(y)$, on which $\varphi$ takes the value $\chi(y)$. In particular

$$
\nabla \varphi(x) \in\left(T_{x} \mathcal{F}_{y}\right)^{\perp}=\mathbb{E} \circ \chi(y)^{\perp}=\mathbb{E} \circ \varphi(x)^{\perp}, \quad \forall x \in \mathcal{F}_{y} .
$$

Since the $\mathcal{F}_{y}$ with $y \in \mathcal{S} \cap B(0, r]$ form a foliation of $B(0, r]$, we have obtained the expected relation (3.10).

Proposition 3.1. Let $\varphi$ be generated by $(\mathbb{E}, \mathcal{S}, \chi)$. The couple $(\varphi, W)$ is well prepared if and only if there exist two functions $W_{\|} \in \mathcal{C}^{1}\left(\mathbb{R}^{2}, \mathbb{R}^{d}\right)$ and $W_{\perp} \in \mathcal{C}^{1}\left(\mathbb{R}, \mathbb{R}^{d}\right)$ satisfying

$$
W_{\|}(t, s) \in \mathbb{E}(t), \quad W_{\perp}(t) \in \mathbb{E}(t)^{\perp}, \quad \forall(t, s) \in \mathbb{R}^{2}
$$

and a scalar function $\psi \in \mathcal{C}^{1}(B(0, r] \times \mathbb{T} ; \mathbb{R})$ such that

$$
W(x, \theta)=W_{\|}(\varphi(x), \psi(x, \theta))+W_{\perp}(\varphi(x)), \quad \forall(x, \theta) \in B(0, r] \times \mathbb{T} .
$$

Proof. Note $\left(Z_{1}(t), \cdots, Z_{J}(t)\right)$ some orthonormal basis of $\mathbb{E}(t)$ with a $\mathcal{C}^{1}$ regularity with respect to $t \in \mathbb{R}$. Complete it with some $\mathcal{C}^{1}$ orthonormal basis $\left(e_{J+1}(t), \cdots, e_{d}(t)\right)$ of $\mathbb{E}(t)^{\perp}$, again of class $\mathcal{C}^{1}$. In view of $(2.9)$ and Lemma 3.2 , the profile $W(x, \theta)$ can be decomposed according to

$$
W(x, \theta)=\sum_{k=1}^{J} w_{j}(x, \theta) Z_{j} \circ \varphi(x)+\sum_{k=J+1}^{d} w_{j}(x) e_{j} \circ \varphi(x)
$$

with

$$
\begin{aligned}
& w_{j} \in \mathcal{C}^{1}(B(0, r] \times \mathbb{T} ; \mathbb{R}), \quad \forall j \in \llbracket 1, J \rrbracket, \\
& w_{j} \in \mathcal{C}^{1}(B(0, r] ; \mathbb{R}), \quad \forall j \in \llbracket J+1, d \rrbracket .
\end{aligned}
$$

Compute the derivative of $W(x, \theta)$ with respect to the variable $x$ and compose on the right with $\Pi_{\nabla \varphi(x)^{\perp}}$. It remains

$$
\begin{aligned}
D_{x} W(x, \theta) \Pi_{\nabla \varphi(x)^{\perp}}= & \sum_{k=1}^{J} \nabla_{x} w_{j}(x, \theta) \cdot \Pi_{\nabla \varphi(x)^{\perp}} \times Z_{j} \circ \varphi(x) \\
& +\sum_{k=J+1}^{d} \nabla_{x} w_{j}(x) \cdot \Pi_{\nabla \varphi(x)^{\perp}} \times e_{j} \circ \varphi(x) .
\end{aligned}
$$

Select a point $(x, \theta) \in \mathcal{V}^{*}$ which means that $\partial_{\theta} W(x, \theta) \neq 0$. Without loss of generality, we can suppose that $\partial_{\theta} W_{J}(x, \theta) \neq 0$. Otherwise, just permute the 
components of $\mathbb{R}^{d}$ to obtain this condition. By construction, the hyperplane $\partial_{\theta} W(x, \theta)^{\perp}$ is generated by the $d-J$ vectors $e_{j} \circ \varphi(x)$ with $j \in \llbracket J+1, d \rrbracket$ and the $J-1$ vectors

$$
\partial_{\theta} w_{J}(x, \theta) Z_{j} \circ \varphi(x)-\partial_{\theta} w_{j}(x, \theta) Z_{J} \circ \varphi(x), \quad j \in \llbracket 1, J-1 \rrbracket .
$$

The requirement (2.16) is equivalent to the conditions

$$
\begin{aligned}
& \nabla_{x} w_{j}(x) \cdot \Pi_{\nabla \varphi(x)^{\perp}}=0, \quad \forall j \in \llbracket J+1, d \rrbracket \\
& \left(\partial_{\theta} w_{J} \nabla_{x} w_{j}-\partial_{\theta} w_{j} \nabla_{x} w_{J}\right)(x, \theta)=0, \quad \forall j \in \llbracket 1, J-1 \rrbracket .
\end{aligned}
$$

On the one hand, from (3.14), we deduce that

$$
\exists \tilde{w}_{j} \in \mathcal{C}^{1}(\mathbb{R}, \mathbb{R}) ; \quad w_{j}(x)=\tilde{w}_{j} \circ \varphi(x), \quad \forall j \in \llbracket J+1, d \rrbracket .
$$

On the other hand, it follows from the relations (3.15) that the mappings $\Upsilon_{t}$ parameterized by $t \in \mathbb{R}$ and defined on the level sets

$$
\mathcal{G}_{t}:=\{x \in B(0, r] ; \varphi(x)=t\}
$$

by the formulas

$$
\begin{aligned}
\Upsilon_{t}: \mathcal{G}_{t} \times \mathbb{T} & \longrightarrow \mathbb{R}^{J} \\
(x, \theta) & \longmapsto{ }^{t}\left(w_{1}, \cdots, w_{J}\right)
\end{aligned}
$$

have rank one. Thus, to each $\Upsilon_{t}$ corresponds a foliation of $\mathcal{G}_{t} \times \mathbb{T}$ by submanifolds of dimension $d-1$. This foliation depends on the parameter $t$. It can be described by using a function $\psi \in \mathcal{C}^{1}(B(0, r] \times \mathbb{T}, \mathbb{R})$ so that

$$
w_{j}(x, \theta)=\tilde{w}_{j}(\varphi(x), \psi(x, \theta)), \quad \forall j \in \llbracket 1, J \rrbracket .
$$

Define

$$
W_{\perp}(t):=\sum_{j=J+1}^{d} \tilde{w}_{j}(t) e_{j}(t), \quad W_{\|}(t, s):=\sum_{j=1}^{J} \tilde{w}_{j}(t, s) Z_{j}(t) .
$$

By construction, we have both (3.12) and (3.13).

Conversely, suppose that $W(x, \theta)$ has the form (3.13) with $W_{\|}(x, \theta)$ and $W_{\perp}(x, \theta)$ as in (3.12). Then

$$
\partial_{\theta} W(x, \theta)=\partial_{\theta} \psi(x, \theta) \times \partial_{s} W_{\|}(\varphi(x), \psi(x, \theta)) \in \mathbb{E}(\varphi(x)) \equiv \mathbf{E}(x)
$$

which is (3.4) and gives rise to the first part of (1.2). Moreover

$$
D_{x} W(x, \theta) \Pi_{\nabla \varphi(x)^{\perp}}=\nabla_{x} \psi(x, \theta) \cdot \Pi_{\nabla \varphi(x)^{\perp}} \times \partial_{s} W_{\|}(\varphi(x), \psi(x, \theta)) .
$$

Since $\partial_{\theta} W$ and $\partial_{s} W_{\|}$are colinear, we get the second equation of (1.2). 


\section{Simple wave solutions}

The aim of this last part is to explain how the initial oscillating data $h^{\varepsilon}(x)$ is transformed through the evolution equation (1). Below, we consider this question in a simplified context, by looking only on simple wave solutions.

Definition 4.1. Let $\varepsilon \in] 0,1]$. We say that $\mathbf{u}^{\varepsilon} \in \mathcal{C}^{1}\left(\Omega^{T} ; \mathbb{R}\right)$ is a simple wave if it can be put in the following form

$$
\mathbf{u}^{\varepsilon}(t, x)=\mathbf{H}\left(t, x, \frac{\Phi(t, x)}{\varepsilon}\right), \quad \mathbf{H} \in \mathcal{C}^{1}\left(\Omega^{T} \times \mathbb{T} ; \mathbb{R}^{d}\right), \quad \Phi \in \mathcal{C}^{1}\left(\Omega^{T} ; \mathbb{R}\right) .
$$

The Theorem 1.1 explains how to associate with a well prepared couple $(\varphi, W)$ a simple wave $\mathbf{u}^{\varepsilon}(t, x)$ which is a solution on $\Omega^{T}$ of the Burger's type system (1). It remains to show this statement 1.1 .

Proof of Theorem 1.1. Compose the first equation of (1.5) with $D_{\mathbf{u}} V \circ \mathbf{H}$ in order to extract

$$
\left\{\begin{array}{l}
\partial_{t} \mathbf{W}+\left(\mathbf{W} \cdot \nabla_{x}\right) \mathbf{W}=0, \\
\partial_{t} \Phi+\left(\mathbf{W} \cdot \nabla_{x}\right) \Phi=0, \\
\mathbf{W}^{*} \cdot \nabla_{x} \Phi=0,
\end{array}\right.
$$

This must be associated with the initial data

$$
\mathbf{W}(0, x, \theta)=W(x, \theta), \quad \Phi(0, x)=\varphi(x) .
$$

First, we discuss about (4.1)-(4.2). From Proposition 3.1 we can write

$$
W(x, \theta)=W_{\|}(\varphi(x), \psi(x, \theta))+W_{\perp}(\varphi(x)) .
$$

Solve locally in time, say on $[0, T] \times B(0, r]$ for some $T>0$, the scalar conservation law

$$
\partial_{t} \Phi+W_{\perp}(\Phi) \cdot \nabla_{x} \Phi=0, \quad \Phi(0, x)=\varphi(x) .
$$

Then, for every $j \in \llbracket 1, J \rrbracket$, we have

$$
\left[\partial_{t}+W_{\perp}(\Phi) \cdot \nabla_{x}\right]\left(Z_{j} \circ \Phi \cdot \nabla_{x} \Phi\right)=-\left(\nabla_{x} \Phi \cdot W_{\perp}^{\prime} \circ \Phi\right) \times\left(Z_{j} \circ \Phi \cdot \nabla_{x} \Phi\right) .
$$

Combining (1.6) and (3.10), we can extract

$$
\left(Z_{j} \circ \Phi \cdot \nabla_{x} \Phi\right)(0, x)=0, \quad \forall(j, x) \in \llbracket 1, J \rrbracket \times B(0, r] .
$$

In view of the preceding equation, this polarization identity is propagated in time which means that

$$
\nabla_{x} \Phi(t, x) \subset \mathbb{E} \circ \Phi(t, x)^{\perp}, \quad \forall(t, x) \in[0, T] \times B(0, r] .
$$


Now, introduce the function

$$
\tilde{W}(t, s):=W_{\|}(t, s)+W_{\perp}(t), \quad(t, s) \in \mathbb{R}^{2}
$$

and the scalar conservation law

$$
\partial_{t} \Psi+\tilde{W}(\Phi(t, x), \Psi) \cdot \nabla_{x} \Psi=0 .
$$

Complete (4.5) with the initial data

$$
\Psi(0, x, \theta)=\psi(x, \theta), \quad \psi \in \mathcal{C}^{1}(B(0, r] \times \mathbb{T} ; \mathbb{R}) .
$$

In (4.5), the variable $\theta \in \mathbb{T}$ plays the part of a parameter. For $T>0$ small enough, the Cauchy problem (4.5)-(4.6) has a local solution on $[0, T] \times$ $B(0, r]$. Finally, define the profile $\mathbf{W}$ through

$$
\mathbf{W}(t, x, \theta):=\tilde{W}(\Phi(t, x), \Psi(t, x, \theta)), \quad \mathbf{W}(0, x, \theta)=W(x, \theta) .
$$

By construction, we have

$$
\mathbf{W}^{*}(t, x, \theta)=W_{\|}(\Phi(t, x), \Psi(t, x, \theta))^{*} .
$$

The informations (3.12) and (4.4) imply that

$$
\mathbf{W}^{*}(t, x, \theta) \cdot \nabla_{x} \Phi(t, x)=0, \quad \forall(t, x) \in[0, T] \times B(0, r] .
$$

Taking into account (3.12) and (4.3), we have also

$$
\partial_{t} \Phi+\mathbf{W} \cdot \nabla_{x} \Phi=\partial_{t} \Phi+W_{\perp} \circ \Phi \cdot \nabla_{x} \Phi=0 .
$$

Then, with (4.5), we can deduce that

$$
\partial_{t} \mathbf{W}+\mathbf{W} \cdot \nabla_{x} \mathbf{W}=\partial_{s} \tilde{W}\left(\partial_{t} \Psi+\mathbf{W} \cdot \nabla_{x} \Psi\right)=0 .
$$

To sum up, the functions $\Phi$ and $\mathbf{W}$ satisfy (4.1). Now, to recover a solution of (1.5), it suffices to solve separately the Cauchy problem

$$
\partial_{t} \mathbf{H}+V \circ \mathbf{H} \cdot \nabla_{x} \mathbf{H}=0, \quad \mathbf{H}(0, x, \theta)=H(x, \theta) .
$$

Define $\mathbf{u}^{\varepsilon}(t, x):=\mathbf{H}\left(t, x, \frac{\Phi(t, x)}{\varepsilon}\right)$ and compute

$$
\begin{aligned}
\partial_{t} \mathbf{u}^{\varepsilon}+V\left(\mathbf{u}^{\varepsilon}\right) \cdot \nabla_{x} \mathbf{u}^{\varepsilon}= & \left(\partial_{t} \mathbf{H}+V \circ \mathbf{H} \cdot \nabla_{x} \mathbf{H}\right)\left(t, x, \frac{\varphi(x)}{\varepsilon}\right) \\
& +\frac{1}{\varepsilon}\left[\left(\partial_{t} \Phi+V \circ \mathbf{H} \cdot \nabla_{x} \Phi\right) \partial_{\theta} \mathbf{H}\right]\left(t, x, \frac{\varphi(x)}{\varepsilon}\right) .
\end{aligned}
$$

The fact that $\mathbf{u}^{\varepsilon}(t, x)$ is a solution of (1) becomes a direct consequence of the equations inside (1.5). Moreover, the definition of $\mathbf{W}$ indicates clealy that the structure (3.13) is conserved for $t \in[0, T]$. Therefore (see the end of the proof of Proposition 3.1), for all $t \in[0, T]$, the trace $(\Phi(t, \cdot), \mathbf{W}(t, \cdot))$ is still well prepared. 


\section{References}

[1] Biryuk, A. On Multidimensional Burgers Type Equations with Small Viscosity , Contributions to current challenges in mathematical fluid mechanics, 1-30, Adv. Math. Fluid Mech., Birkhäuser, Basel (2004).

[2] Cheverry, C. Recent results in large amplitude monophase nonlinear geometric optics, to appear in the International Mathematical Series, Series Ed.: Rozhkovskaya,Tamara, Springer, Vols 5/6, 21 p.

[3] Cheverry, C. ; Guès, O. Counter-examples to concentration-cancellation and supercritical nonlinear geometric optics for the incompressible Euler equations, to appear in Archive for Rational Mechanics and Analysis, Springer, $72 \mathrm{p}$.

[4] Gallagher, I. ; Saint-Raymond, L. On pressureless gases driven by a strong inhomogeneous magnetic field, SIAM J. Math. Anal. 36 (2005), no. $4,1159-1176$.

[5] Majda, Andrew J. Compressible fluid flow and systems of quasilinear equations in several space variables, Applied Mathematical Sciences, vol 53, Springer-Verlag, New York (1984).

[6] Poupaud, F. Global smooth solutions of some quasi-linear hyperbolic systems with large data, Ann. Fac. Sci. Toulouse Math. (6) 8, no. 4, 649-659 (1999). 